PROCEEDINGS OF THE FIFTH INTERNATIONAL SEMINAR ON RRDPAE-2002

"Recent Research and Design Progress in Aeronautical Engineering and its Influence on Education" Vilnius - Lithuania

\title{
SCALED HIGH ANGLE RESEARCH VEHICLE SHARV) PROGRAM
}

\section{Szender}

Warsaw University of Technology.E-mail: mszender@yahoo.com Received 2711 2002, accepted 26022004

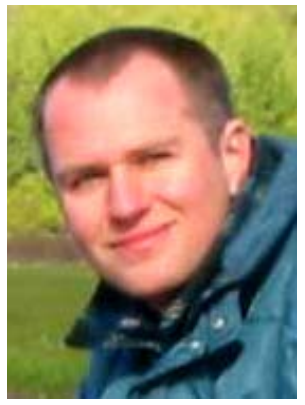

Marcin SZENDER was born 1972. MSc in aircraft engineering from Warsaw University of Technology 1998. Phd student at WUT - FPAE. "Bielik" aircraft designing team member since 1999

\begin{abstract}
A flight test research program employing a remotely piloted vehicle (RPV) within high angle of attack range has commenced at the Faculty of Power and Aeronautical Engineering of Warsaw University of Technology, Poland. The initial flights of the scaled model of the "Bielik" aircraft were made with the aim to correlate RPV and full-scale flight stall and departure and spin controllability considering the effects of dynamic scaling laws and Reynolds number. The remotely controlled research aircraft, which is powered by a turbine jet engine and equipped with a flight data recording system, proved to be good source of stability and control data at relatively low cost and without additional risk. The research remotely piloted vehicle and results of its initial flights are presented in this paper.
\end{abstract}

Keywords: flight tests, dynamically scaled models, high angle of attack.

\section{Introduction}

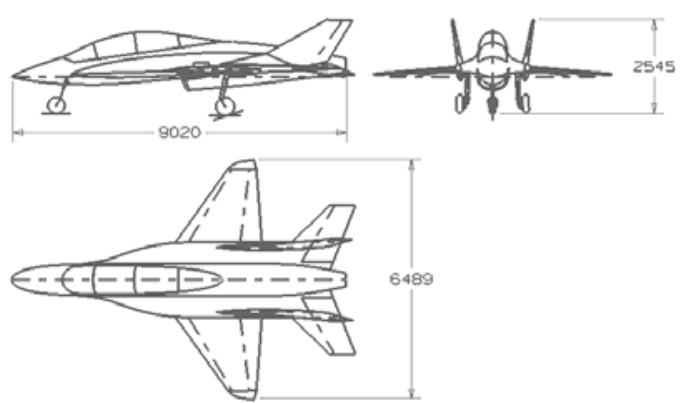

Fig 1. "Bielik" layout

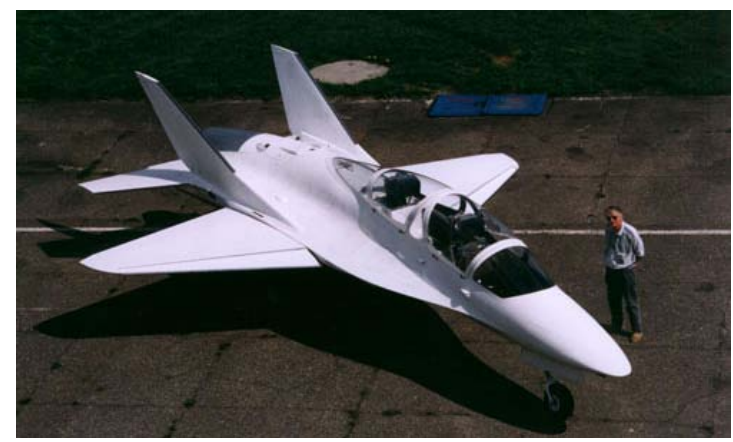

Fig 2. "Bielik" aircraft
"Bielik" is a full composite, subsonic jet training aircraft designed and built at Edward Marganski Aircraft Workshop Bielsko-Biała, Poland. Designed as low-cost airplane for the advanced training of pilots. The first prototype is currently undergoing preflight tests.

\section{Research Aircraft Description}

The Scaled High Angle Research Vehicle (SHARV) is a dynamically scaled, remotely piloted model of "Bielik". It has full composite structure, including a high temperature resin system in the aft part of the fuselage around the turbine exhaust section. Because of the fact that the same airframe was used for both flight and wind tunnel tests, the SHARV airframe was designed for load factors $+15 /-7$. The research aircraft is powered by a single Jet Cat P120 jet turbine engine generating 120 Newton's of thrust. The aircraft is equipped with a pneumatically activated landing gear with brakes installed on the main wheels. It is remotely piloted by a 12 channel pulse code modulation system which controls the following functions: elevens, rudders, leading and trailing edge flaps, turbine RPM, landing gear retraction, and main wheels breaks. It may also activate the emergency rescue parachute. 
SHARV main dimensions and weights:

$\begin{array}{lcc}\text { Length } & 1.9 \mathrm{~m} & \\ \text { Span } & & 1.3 \mathrm{~m} \\ \text { Empty weight } & & 12 \mathrm{~kg} \\ \text { TTO weight } & 17 \mathrm{~kg} & \end{array}$

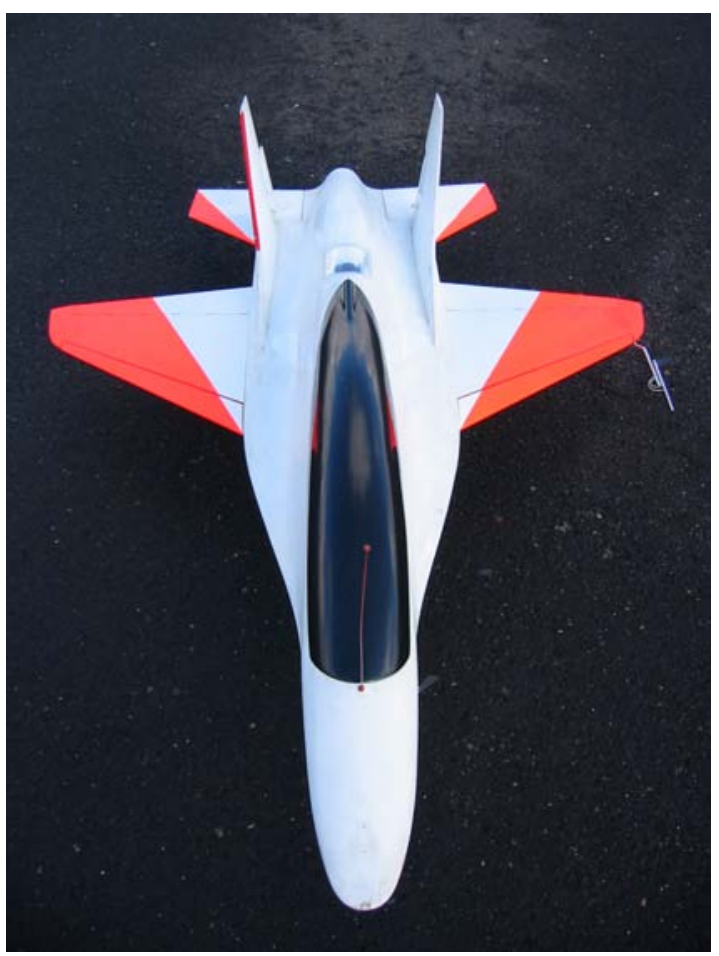

Fig 3. SHARV

\section{Goals of the SHARV flight-test program}

A flight test program employing a scale model gives opportunities to obtain airplane flight characteristic at a lower cost and without risk before the full-scale airplane makes it first flight. The following high angle of attack phenomena are planned to be investigated during this program:

- Stability and controllability at high angle of attack. Stability with different SG position will be investigated joined with visualization of forebody/strake and leading edge vortex flow

- Spin characteristics. Spin resistance of configuration will be checked. Spin modes at high and medium high angle of attack will be identified and recovery method will be investigated

- Analysis of method employing dynamically scaled models within high angle of attack range.

All results obtained from the SHARV flight test will be transferred to full-scale aircraft characteristics.

\section{Scaling theory}

The SHARV is one-twentieth the size of the "Bielik" aircraft. All requirements of dynamic scaling are fulfilled according to the following relations:
$\lambda_{\mathrm{L}}=\mathrm{RPV} \operatorname{dim} /$ Full-scale Aircraft $\operatorname{dim}$ then

\begin{tabular}{|l|l|}
\hline Scale & $\lambda_{\mathrm{L}}$ \\
\hline Linear Dimension & $\lambda_{\mathrm{L}}$ \\
\hline Area & $\lambda^{2}{ }_{\mathrm{L}}$ \\
\hline Mass & $\lambda_{\mathrm{L}}^{3}$ \\
\hline Force & $\lambda_{\mathrm{L}}^{3}$ \\
\hline Moment of Force & $\lambda_{\mathrm{L}}^{4}$ \\
\hline Moment of Inertia & $\lambda_{\mathrm{L}}^{5}$ \\
\hline Time & $\lambda^{0.5}{ }_{\mathrm{L}}$ \\
\hline Linear Velocity & $\lambda^{0.5}{ }_{\mathrm{L}}$ \\
\hline Angular Velocity & $\lambda^{-0.5} \mathrm{~L}$ \\
\hline Linear Acceleration & 1 \\
\hline Angular Acceleration & $\lambda^{-1}{ }_{\mathrm{L}}$ \\
\hline Work & $\lambda_{\mathrm{L}}^{4}$ \\
\hline Power & $\lambda^{3.5}{ }_{\mathrm{L}}$ \\
\hline Lift Coefficient- $\mathrm{C}_{1}$ & 1 \\
\hline Drag Coefficient- $\mathrm{C}_{\mathrm{d}}$ & 1 \\
\hline
\end{tabular}

The research program is focused at the higher angle of attack range (above the linear range of $\mathrm{C}_{\mathrm{L}}$ vs. AOA characteristic-Fig 4.), which is Reynolds number independent. Even though SHARV and "Bielik" have different linear dimensions and, consequently, different Reynolds Numbers, as long as a high angle of attack range is considered, aerodynamic similarity is also fulfilled.

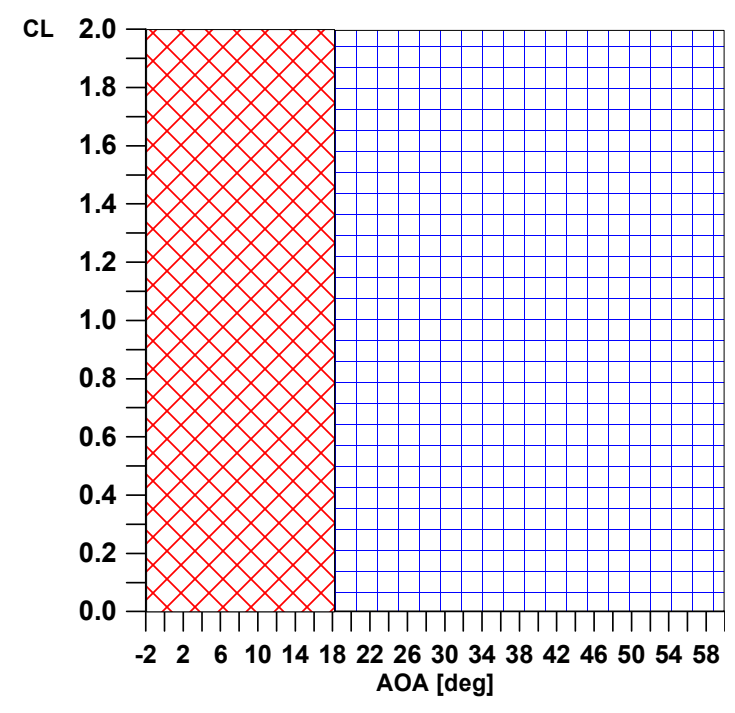

Fig 4. $\mathrm{C}_{\mathrm{L}}$ vs. AOA

\section{Flight Data Recording System}

A 20 channel, $50 \mathrm{~Hz}$ sampling frequency data recording device is installed on board the SHARV. The unit was specially designed by the Air Force Institute of Technology in Warsaw for flight tests of scaled airplanes. It measures and records the following values:

- Indicated Air Speed

- $\quad$ Altitude

- Angle of attack "mechanical method" 
- Angle of attack "pressure method"

- $\quad$ Angle of slip

- Turbine RPM

- Linear Accelerations: Ax, Ay, Az

- Angular velocity: $\Omega_{\mathrm{X}}, \Omega_{\mathrm{y}}, \Omega_{\mathrm{z}}$

- $\quad$ Elevens deflection: L and R

- $\quad$ Rudders deflection $\mathrm{L}$ and $\mathrm{R}$

- $\quad$ Trailing Edge Flaps Position

- Leading Edge Flaps Position

- $\quad$ GPS Speed

- GPS

AltitudeGPS

Position

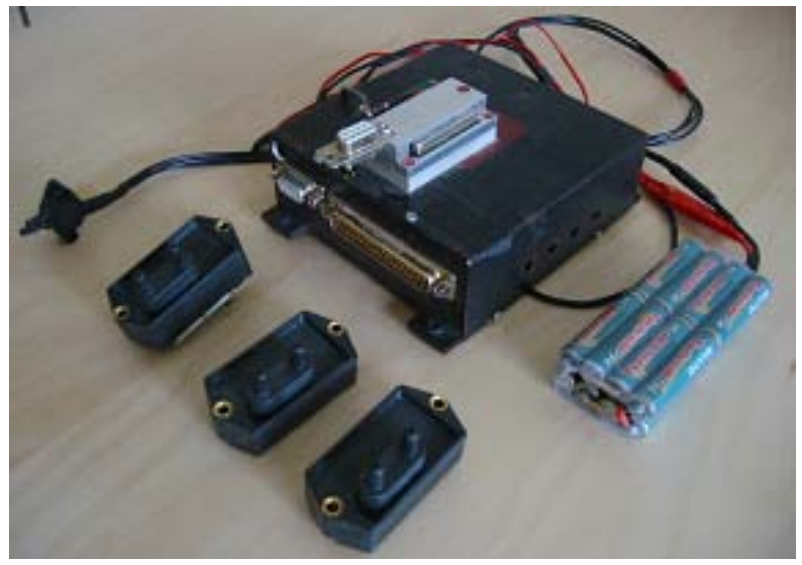

Fig 5. Flight data recorder

SHARV is also equipped with two micro video cameras connected with an onboard transmitter. This visualization system allows two views on the ground to be observed in real time. The views chosen depend on the analyzed flight task and may include:

- $\quad$ Front view (pilot view)

- Rear view

- Forebody/strake vortex flow visualization on both sides of the aircraft

\section{Method of measuring angle of attack}

Two different methods of measuring angle of attack (AOA) were employed. The first "classic" method was based on the rotating vane adjusting its position to the actual flow vector. The accuracy of this method, which was verified in the wind tunnel, was $+/-1$ deg at stationary flight modes but at a high pitch rate, inertial moments generated unacceptable indication errors. These errors forced the development of the method of indicating AOA based on the difference in pressure measured between the top and bottom of the nose cone of the research aircraft. Wind tunnel tests showed that for angels of attack ranging from -15 to 50 degrees, the pressure difference between top and bottom is linear function of AOA, and its slope depends on dynamic pressure.

This system, which was developed on a smaller wind tunnel model, was installed on the SHARV and calibrated during wind tunnel tests. The accuracy of the "pressure" method was $+/-1$ deg.

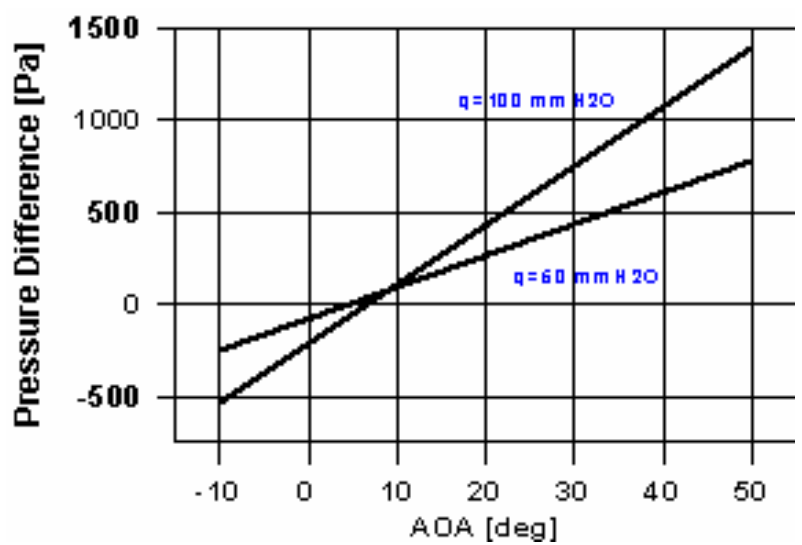

Fig 6. Pressure difference between top and bottom at the aircraft nose as a function of angle of attack

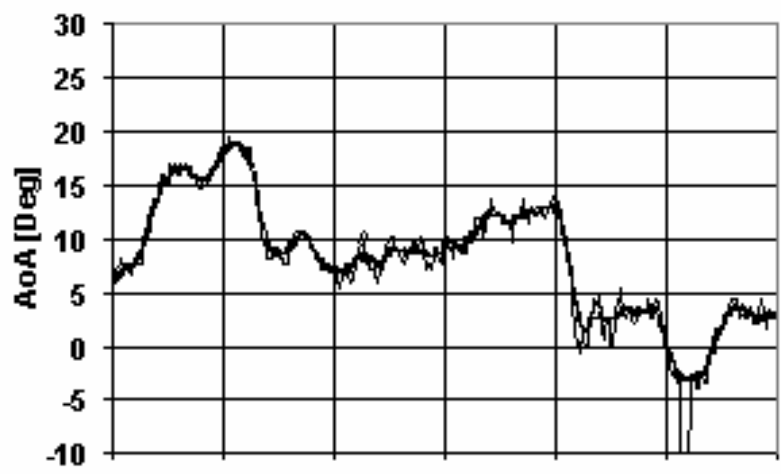

Flight Time

Fig 7. Comparison of methods of measuring AoA

Comparisons of methods of measuring mechanical and pressure AOA (Fig 7.) showed fair correlation and the only differences are generated by the mechanical method in non-stationary flight modes.

\section{Wind Tunnel Tests}

Before flight tests were started, some wind tunnel tests were done. $\varnothing 5 \mathrm{~m}$ wind tunnel at the Institute of Aviation in Warsaw was used. We obtained aerodynamic characteristics and calibration of the system measuring angle of attack and angle of slip.

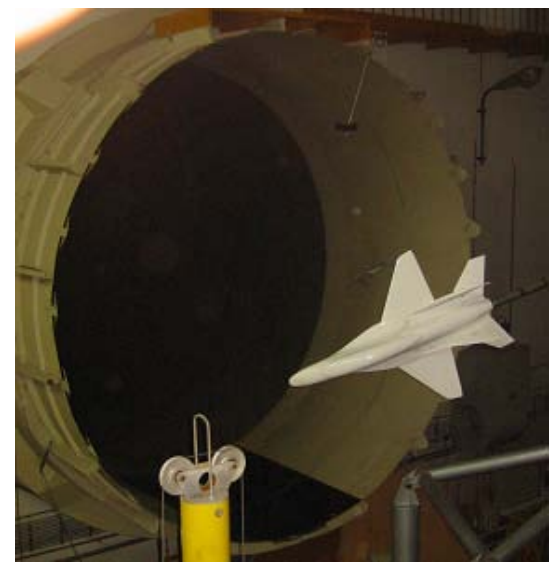

Fig 8. SHARV in $Ø 5 \mathrm{~m}$ wind tunnel at the Institute of Aviation in Warsaw 


\section{Flight Tests Results}

A typical research flight mission is presented in figures 9,10 and $11 \ldots$ The figures represent barometric altitude, indicated air speed, and aircraft position based on the GPS. Figures 12 to 17 presents' aircraft stall with the engine at idle. Recorded stall air speed dropped almost to $0 \mathrm{~m} / \mathrm{s}$ and $200 \mathrm{~m}$ of altitude decrease can be observed. Linear accelerations in $\mathrm{X}$ and $\mathrm{Z}$ directions and left and right elevens recorded during stall are presented in figures 14 to 17 .

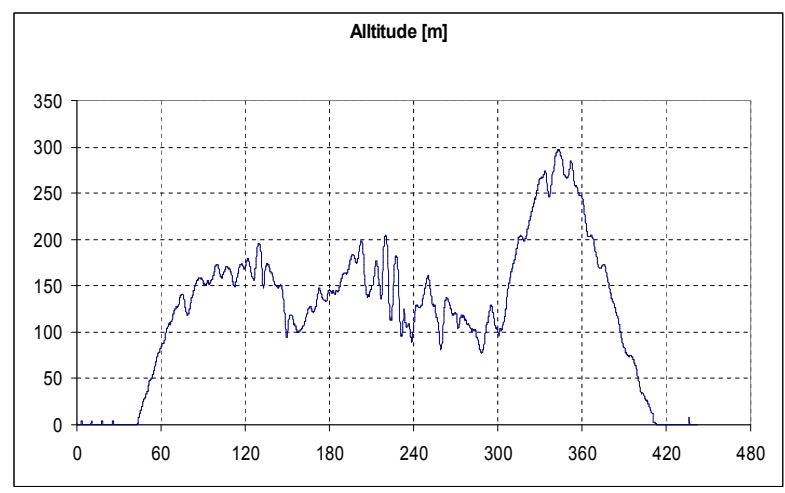

Fig 9. Flight mission altitude in [m]

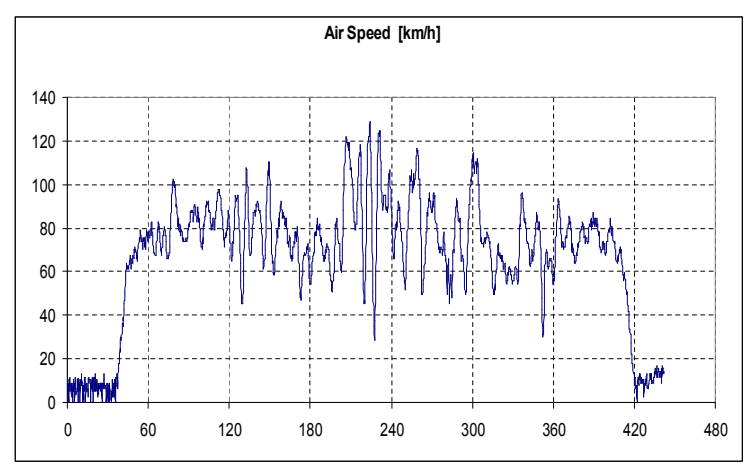

Fig 10. Flight mission air speed in $[\mathrm{km} / \mathrm{h}]$

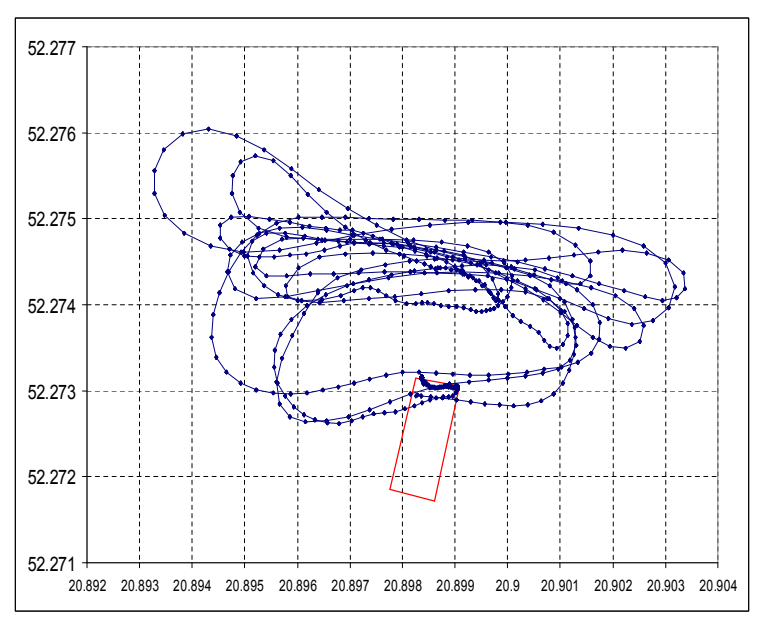

Fig 11. Flight aircraft position

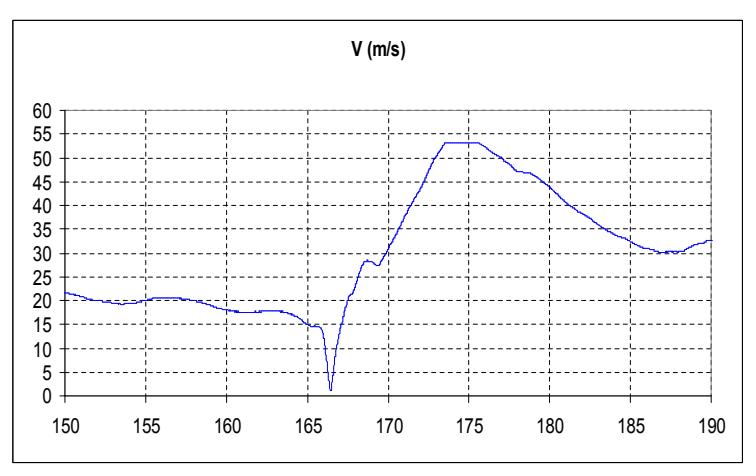

Fig 12. Stall air speed in $[\mathrm{m} / \mathrm{s}]$

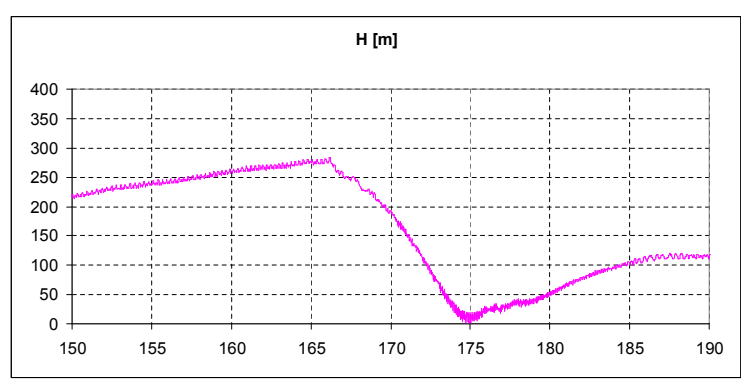

Fig 13. Stall altitude in $[\mathrm{m}]$

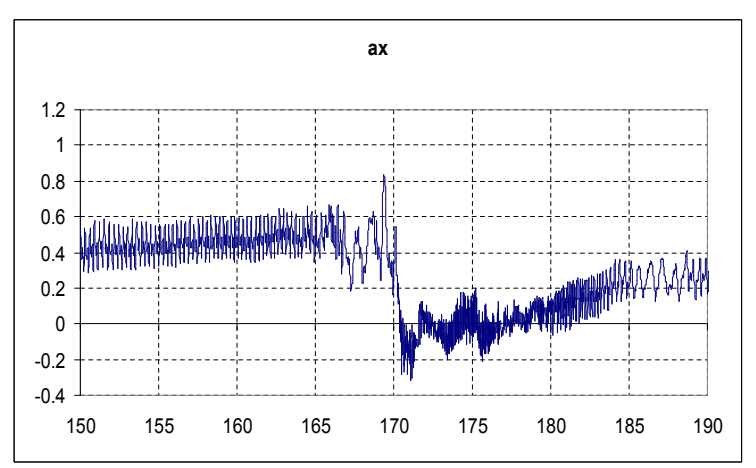

Fig 14. Stall linear acceleration in $\mathrm{X}$ direction

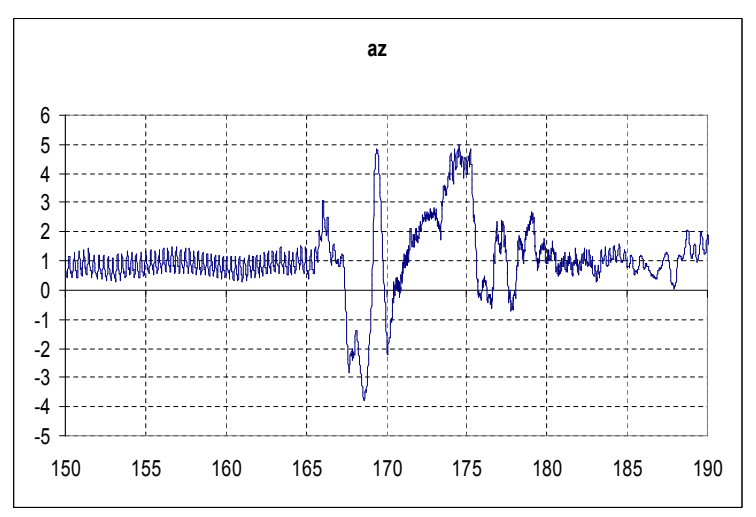

Fig 15. Stall linear acceleration in $\mathrm{Z}$ direction 


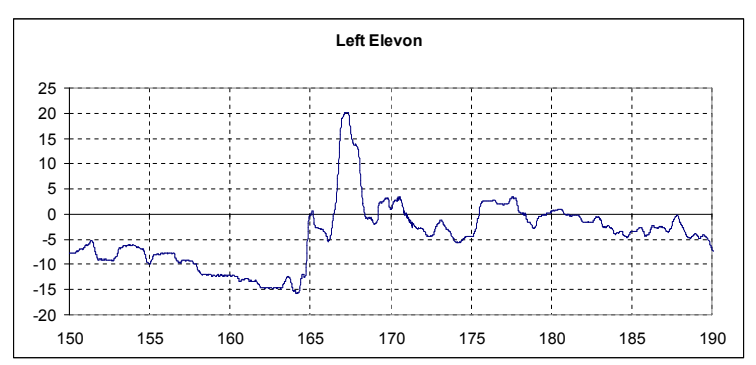

Fig 16. Left elevon deflection [deg]

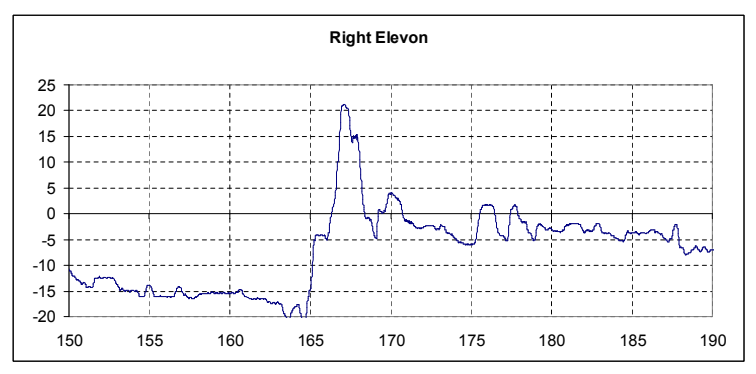

Fig 17. Right elevon deflection $[\mathrm{deg}]$

\section{Conclusions}

During the initial flights of the SHARV, we were not able to define the flight characteristics of the aircraft, but results obtained from these flights shows that remotely piloted vehicle, equipped with the same (scaled) flight control laws as the full scale aircraft could bring added safety to the flight test program, improving the test conditions for the test pilots and limiting some of the financial risks.

\section{References}

1. Croom M., Kenney H., D. Murri D. et al. Research on the F/A-18E/F Using a 22 Percent-DynamicallyScaled Drop Model // AIAA Atmospheric Flight Mechanics Conference and Exhibit, Denver, Colorado.

2. Fisher D. F., Del Frate J.H., Richwine D. In-flight flow visualization characteristics of the NASA f-18 high alpha research vehicle at high angles of attack // NASA-TM-4193. - May 1991.

3. Fisher D.F., Banks D.W., Richwine D.M. F-18 high alpha research vehicle surface pressures: initial inflight results and correlation with flow visualization and wind-tunnel data // NASA-TM-101724.

4. Holleman E.C. Summary of flight tests to determine the spin and controllability characteristics of a remotely piloted, large-scale (3/8) fighter airplane model // NASA-TN-D-8052.

5. Moes T.R., Whitmore S.A. A preliminary look at techniques used to obtain air data from flight at high angles of attack // Technical Memorandum, NASATM-101729. - December 1990. 